\title{
Fibrolamellar carcinomas are positive for CD68
}

\author{
Hillary M Ross ${ }^{1}$, Hubert DJ Daniel ${ }^{1}$, Perumal Vivekanandan ${ }^{1}$, Rajesh Kannangai ${ }^{1}$, \\ Matthew M Yeh ${ }^{2}$, Tsung-Teh $\mathrm{Wu}^{3}$, Hala $\mathrm{R}$ Makhlouf ${ }^{4}$ and Michael Torbenson ${ }^{1}$ \\ ${ }^{1}$ Department of Pathology, The Johns Hopkins University School of Medicine, Baltimore, MD, USA; \\ ${ }^{2}$ Department of Pathology, University of Washington, Seattle, WA, USA; ${ }^{3}$ Department of Pathology, \\ Mayo Clinic, Rochester, MN, USA and ${ }^{4}$ Armed Forces Institute of Pathology, Washington, DC, USA
}

\begin{abstract}
Fibrolamellar carcinomas are a unique type of liver carcinoma that arise in non-cirrhotic livers of young individuals. Despite their distinctive appearance, recent studies have demonstrated a lack of consistency in how fibrolamellar carcinomas are diagnosed by pathologists. As a potential aide in diagnosis, we investigated the staining properties of CD68. The CD68 gene encodes for a transmembrane glycoprotein located within lysosomes and endosomes. Macrophages as well as other cell types rich in lysosomes/endosomes are CD68 positive. Cases of fibrolamellar carcinoma were collected from four academic centers. Control groups included hepatocellular carcinomas arising in both non-cirrhotic livers and cirrhotic livers. A group of cholangiocarcinomas were also stained. CD68 immunostaining was scored for both intensity and distribution on a scale of 0 to $3+$. Twenty-three primary fibrolamellar carcinomas and 9 metastases (total of 24 individuals) were immunostained and showed a distinctive granular, dot-like or stippled pattern of cytoplasmic staining in nearly all cases (31/32), with a median distribution and intensity score of $3+$. In control hepatocellular carcinomas that arose in non-cirrhotic livers, 10/39 showed CD68 staining with a median distribution and intensity score of $2+$. In hepatocellular carcinomas arising in cirrhotic livers, 3/27 cases showed CD68 positivity, all with stippled dot-like cytoplasmic staining similar to that of fibrolamellar carcinomas. All five cholangiocarcinomas were negative. Overall, CD68 positivity was strongly associated with fibrolamellar carcinomas, $P<0.001$ and had a sensitivity of $96 \%$, a specificity of $80 \%$, and a negative predictive value of $98 \%$. In sum, tumor positivity for CD68 staining was highly sensitive for fibrolamellar carcinoma and a lack of CD68 staining should suggest caution in making a diagnosis of fibrolamellar carcinoma.
\end{abstract}

Modern Pathology (2011) 24, 390-395; doi:10.1038/modpathol.2010.207; published online 26 November 2010

Keywords: CD68; cholangiocarcinoma; fibrolamellar carcinoma; hepatocellular carcinoma

Fibrolamellar carcinomas are a unique type of liver carcinoma that arise in non-cirrhotic livers of young individuals. ${ }^{1}$ Their etiology is unknown. Histologically, the tumor is made up of large polygonal cells with abundant eosinophilic cytoplasm, large vesiculated nuclei, and large nucleoli. Lamellar band of fibrosis are present within the tumor and can be seen in both primary and metastatic tumors.

Despite their distinctive appearance, there can be a lack of consistency in how fibrolamellar carcinomas are diagnosed by pathologists. Even among experts, weak reproducibility was seen in the histological diagnosis of primary liver tumors with

Correspondence: Dr M Torbenson, MD, Department of Pathology, Johns Hopkins University School of Medicine, 1503 East Jefferson, Room B314, Baltimore, MD 21231, USA.

E-mail: mtorben@jhmi.edu

Received 01 July 2010; revised 29 August 2010; accepted 30 August 2010; published online 26 November 2010 fibrous stroma in non-cirrhotic patients and a consensus diagnosis was achieved in only $32 \%$ of the cases. ${ }^{2}$ As another example, a large difference is seen in the average age of diagnosis for cases of fibrolamellar carcinoma published in the peerreviewed literature versus cases diagnosed in the SEER database, ${ }^{3}$ a difference most likely explained by inconsistencies in making the diagnosis of fibrolamellar carcinoma. Histologically, a common diagnostic pitfall is the overinterpretation of intratumoral fibrosis as being diagnostic of fibrolamellar carcinoma. In addition, in our experience, another common reason for misdiagnosis is a misperception that most cases of primary liver carcinoma in children and young adults are fibrolamellar carcinomas, leading to over diagnosis of fibrolamellar carcinoma in this age group, especially with tumors arising in livers without underlying disease.

We have previously explored the role for immunostain markers as an aide in the diagnosis 
of fibrolamellar carcinomas ${ }^{4,5}$ but sought for additional markers among the currently used panel of antibodies available in most hospital laboratories. Further analysis of previously reported gene expression $^{6}$ studies showed a modest increase in the expression of CD68. The CD68 gene encodes for a transmembrane glycoprotein located within lysosomes and endosomes. Thus, macrophages as well as other cell types rich in lysosomes/endosomes are CD68 positive. In this study, the staining pattern of CD68 was explored in the epithelial cells of both fibrolamellar and typical hepatocellular carcinomas.

\section{Materials and methods}

\section{Tissues}

Archival cases were collected from four academic centers (Mayo Clinic, University of Washington, Armed Forces Institute of Pathology, and Johns Hopkins Hospital). A total of 23 primary fibrolamellar carcinomas and nine metastatic fibrolamellar carcinomas were studied (Table 1). The tumors were from 24 individuals. Eight of the nine metastases had a paired primary fibrolamellar available to study. The large number of metastatic cases were intentionally selected to investigate the staining properties in metastatic tumors. The metastases were all extrahepatic.

To be sure of the appropriate diagnosis of fibrolamellar carcinomas, only full sections from completely resected tumors were evaluated and all cases were centrally reviewed. The histological diagnoses were confirmed in all cases by routine light microscopy. To allow the use of normal liver tissues as internal slide controls, sections of the interface of the tumor and non-tumor tissues were used whenever possible.

Tissues from 66 individuals with typical hepatocellular carcinoma were also studied (Table 1) as controls. Twenty-seven cases arose in cirrhotic livers while 39 cases arose in non-cirrhotic livers. Five individuals with cholangiocarcinomas were also included as a control group. For all control cases, only full sections of tumor were used.

Table 1 Demographical findings

\begin{tabular}{|c|c|c|c|}
\hline Tumor & Cases & $\begin{array}{l}\text { Age } \pm \text { s.d } \\
\text { (years) }\end{array}$ & Gender \\
\hline Fibrolamellar carcinoma & $32^{\mathrm{a}}$ & $32 \pm 14$ & $8 \mathrm{M}: 16 \mathrm{~F}$ \\
\hline $\begin{array}{l}\text { Hepatocellular carcinoma cirrhotic } \\
\text { background liver }\end{array}$ & 27 & $58 \pm 14$ & 17M:9F \\
\hline $\begin{array}{l}\text { Hepatocellular carcinoma } \\
\text { non-cirrhotic background liver }\end{array}$ & 39 & $60 \pm 15$ & $21 \mathrm{M}: 18 \mathrm{~F}$ \\
\hline
\end{tabular}

${ }^{\mathrm{a}}$ Includes 23 primary and 9 metastatic cases from a total of 24 individuals.

\section{Immunohistochemistry}

Cases were stained and scored at a central location (JHU). A pilot study of five cases and controls was initially performed using the hospital laboratory and, when successful, the study was continued using manual staining because of cost considerations. The KP1 clone antibody was used (Ventana, catalog number 790-2931 for autostainer; Dako, catalog number M0814 for manual staining) and both performed equally well. CD68 immunostaining was scored for both intensity and distribution on a scale of 0 to $3+$. A score of 1 for distribution indicated only patchy positivity, with 5 to $30 \%$ of tumor cells showing cytoplasmic positivity; a score of 2 represented $\sim 31$ to $60 \%$ of cells positive; and a score of 3 represented $>60 \%$ of tumor cells were positive. A score of 1 for intensity represented clear cytoplasmic positivity only seen at high power ( $\times 40$ lens); a score of 2 represented positivity seen at medium power $(\times 20$ lens); and a 3 represented positivity visible at low power $(\times 4$ lens).

To better understand if the CD68 positivity by immunostaining reflected increased gene expression, versus concentration of lysosomal proteins due to the numerous mitochondria in the cytoplasm of fibrolamellar carcinomas, mRNA expression was measured in a set of seven fibrolamellar carcinomas from Johns Hopkins Hospital with available frozen tissue. A comparison group of six typical hepatocellular carcinomas arising in the setting of hepatitis $\mathrm{C}$ and liver cirrhosis were also studied. All tissues were frozen in liquid nitrogen and stored at $-80^{\circ} \mathrm{C}$ prior to use. The pathological diagnoses were confirmed in all cases. Total RNA was extracted using TRIzol (Invitrogen Life Technologies, Carlsbad, CA, USA) followed by precipitation with isopropyl alcohol. cDNA was prepared as per the manufacturer's instructions using $1 \mu \mathrm{g}$ of RNA with oligo dT primers and the Superscript First-Strand synthesis system for RT-PCR (Invitrogen Life Technologies). CD68 Primers were designed to span across an intron: $\mathrm{F}$ 5'-ACGCAGCAcaGTGGACATT3'; R 5'-GTCACTGGGGCAGGAGAAAC-3'. Real-time PCR was performed using the SmartCycler system (Cepheid) with Fast Start SYBR green master mix (Roche) with $2 \mu \mathrm{g}$ of cDNA and cycling conditions of $95^{\circ} \mathrm{C}$ for $10 \mathrm{~min}$ followed by 40 cycles of $95^{\circ} \mathrm{C}$ for $18 \mathrm{~s}, 57^{\circ} \mathrm{C}$ for $20 \mathrm{~s}$, and $72^{\circ} \mathrm{C}$ for $30 \mathrm{~s}$. The specificity of PCR products was ascertained by melt curve analysis and by size determination in agarose gel. Real-time PCR was negative when performed on cDNA prepared without reverse transcriptase. Expression levels were normalized to $\beta$-glucuronidase. As most CD68 expression in the non-neoplastic liver is from Kupffer cells, comparing level of CD68 in tumors versus non-tumor does not make good biological sense. For that reason, CD68 levels for each tumor were normalized to a house keeping gene using relative curves and the ratio of CD68 to $\beta$-glucuronidase calculated. ${ }^{7}$ 


\section{Results}

\section{Immunohistochemistry}

Twenty-three primary fibrolamellar carcinomas and nine metastases (total of 24 individuals) were studied. All cases showed the typical histological findings of fibrolamellar carcinoma (Figure 1a) and all cases arose in livers with no known underlying liver disease. When stained with CD68, nearly all cases (31/32) were positive, with most showing a distinctive granular or stippled pattern. The median score for both the distribution and intensity was $3+$ for fibrolamellar carcinoma cases (Figure 1b and c). The background liver tissues showed strong diffuse staining of Kupffer cells, with no significant staining of hepatocytes (Figure 1d). This served as a critical internal control. The weakest staining fibrolamellar carcinomas showed $1+$ intensity and $3+$ distribution, which was seen in two cases (Figure 1e). In two cases, the fibrolamellar carcinomas showed a somewhat different staining pattern, with one or two larger cytoplasmic 'dots' instead of the granular or stippled staining pattern (Figure 1f). The dot-like positivity did not appear to correlate with the presence of pale bodies. The reason(s) for the lack of staining in the one negative fibrolamellar carcinoma case is unclear. A re-review of the histological features confirmed it to be a fibrolamellar carcinoma.

In control hepatocellular carcinomas that arose in non-cirrhotic livers, 10/39 cases showed CD68 staining, with a median score for both distribution and intensity of $2+$ (Figure 2). In 4 of these 10 cases, there was a stippled dot-like cytoplasmic staining similar to fibrolamellar carcinomas (Figure 2a). Several of these cases had a fatty morphology (Figure 2b). The remaining six positive cases showed a more diffuse weak-to-moderate cytoplasmic positivity (Figure 2c). In hepatocellular carcinomas arising in cirrhotic livers, $3 / 27$ cases showed CD68 positivity. These three cases each showed a stippled dot-like cytoplasmic staining similar to that of fibrolamellar carcinomas. In one of these cases, the CD68 staining was seen in proximity to tumor necrosis (Figure 2c). Careful inspection of the morphology of the staining cells in this case indicated that viable tumor cells were staining. All five cholangiocarcinomas were negative.

Overall, CD68 positivity was strongly associated with fibrolamellar carcinomas, $P<0.001, \chi^{2}$ test. CD 69 positivity had a sensitivity of $96 \%$, a specificity of $80 \%$, and a negative predictive value of $98 \%$ for diagnosing fibrolamellar carcinoma (Table 2). Typical hepatocellular carcinomas arising in non-cirrhotic livers had a higher frequency of CD68 staining $(10 / 39 ; 26 \%)$ than those arising in cirrhotic livers $(3 / 27 ; 11 \%)$, although the $P$-value was not significant, $P=0.14$, $\chi^{2}$ test.
By real-time PCR, CD68 mRNA was overexpressed in fibrolamellar carcinomas compared to typical hepatocellular carcinomas, $P=0.008$, Student's $t$-test (Figure 3).

\section{Discussion}

Despite the distinctive clinical and histological features of fibrolamellar carcinoma, they can be a diagnostic challenge. To improve the reproducibility of the histological diagnosis, we have searched over the past several years for immunohistochemical stains that may be of assistance. In one approach, we showed that fibrolamellar carcinomas are routinely positive for CK7, while only about one third of typical hepatocellular carcinomas are CK7 positive. ${ }^{5}$ This overall pattern of strong CK7 positivity in fibrolamellar carcinomas has recently been confirmed by two additional groups of investigators. ${ }^{8,9}$ In searching for a more specific marker for fibrolamellar carcinomas, we have shown that anterior gradient two is overexpressed in most cases, but is only rarely expressed in typical hepatocellular carcinomas. ${ }^{4}$ In this current study, we further extend the immunohistochemical profile of fibrolamellar carcinomas to include CD68 staining. Because of the routine availability of CD68 in most hospital laboratories, we believe this approach can be helpful in routine surgical practice. Our study design further enhances the applicability of this approach, as we have shown CD68 staining among cases from four different institutions and have also shown that metastatic tumors do not lose their CD68 positivity.

Interpretation of CD68 staining in this study was greatly facilitated by the use of sections from the interface of the tumor and normal. The nonneoplastic liver on the slides showed diffuse and strong CD68 staining in Kupffer cells. Any cases that lacked such staining were repeated. Also of note, we did not include any biopsies. Only full sections were included as part of the study design, as this allowed the greatest assurance of the proper diagnosis. In addition, all blocks were freshly cut for this study (ie archival sections were not studied). Using this careful approach, this study demonstrates a distinctive pattern of immunostaining for CD68 in fibrolamellar carcinomas. Nearly all cases showed a granular, stippled pattern, or a dot-like pattern of positivity. Based on this multi-institutional series of cases, this staining pattern is highly sensitive for fibrolamellar carcinomas, but is not specific. In terms of its possible role in routine clinical use, given its high negative predictive value, a diagnosis of fibrolamellar carcinoma in a primary liver carcinoma that is CD68 negative should be strongly re-considered to ensure that fibrolamellar carcinoma is the appropriate diagnosis.

In addition to the fibrolamellar carcinomas, this distinctive pattern was also seen in a minority of 

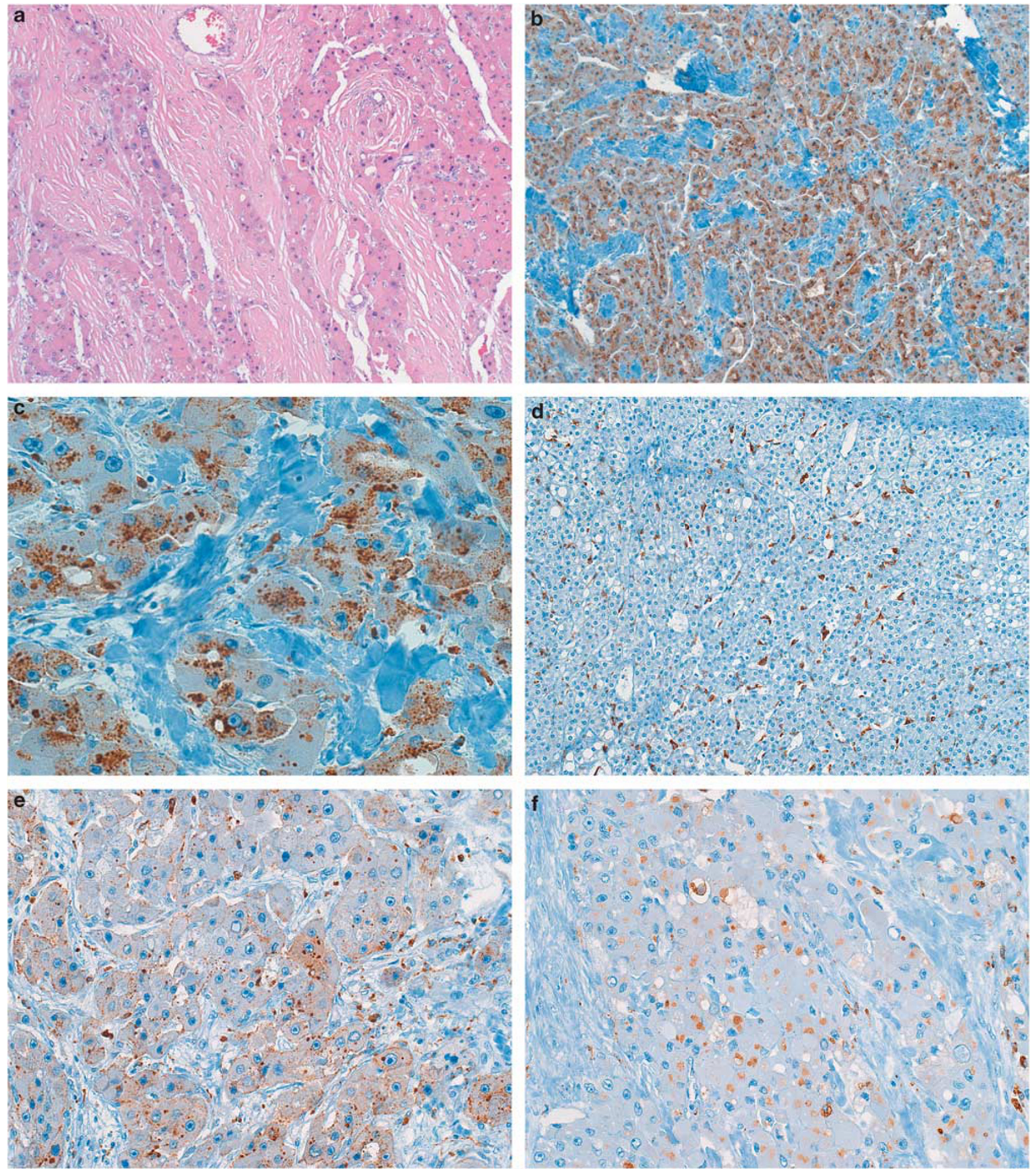

Figure 1 Fibrolamellar carcinomas are CD68 positive. (a) H\&E, original magnification, $\times 40$. The typical morphology of fibrolamellar carcinoma can be seen. (b) CD68, original magnification, $\times 40$ : fibrolamellar carcinomas show a distinctive granular or stippled cytoplasmic staining pattern. (c) CD68, original magnification, $\times 160$ : High-power image of CD68 staining in fibrolamellar carcinoma. (d) CD68, original magnification, $\times 40$ : the background liver (same case as b, c) shows CD68 staining in Kupffer cells, but not the hepatocytes. (e) CD68, original magnification, $\times 100$ : another case of fibrolamellar carcinoma showing weaker but still positive CD68 staining. (f) CD68, original magnification, $\times 100$ : in some cases of fibrolamellar carcinoma, the tumor showed a larger circumscribed dotlike staining pattern of CD68.

conventional hepatocellular carcinoma in both of our controls groups of hepatocellular carcinomas arising in cirrhotic and non-cirrhotic livers.
Although not statistically significant, a larger percentage of conventional hepatocellular carcinomas that arose in non-cirrhotic livers than cirrhotic livers 

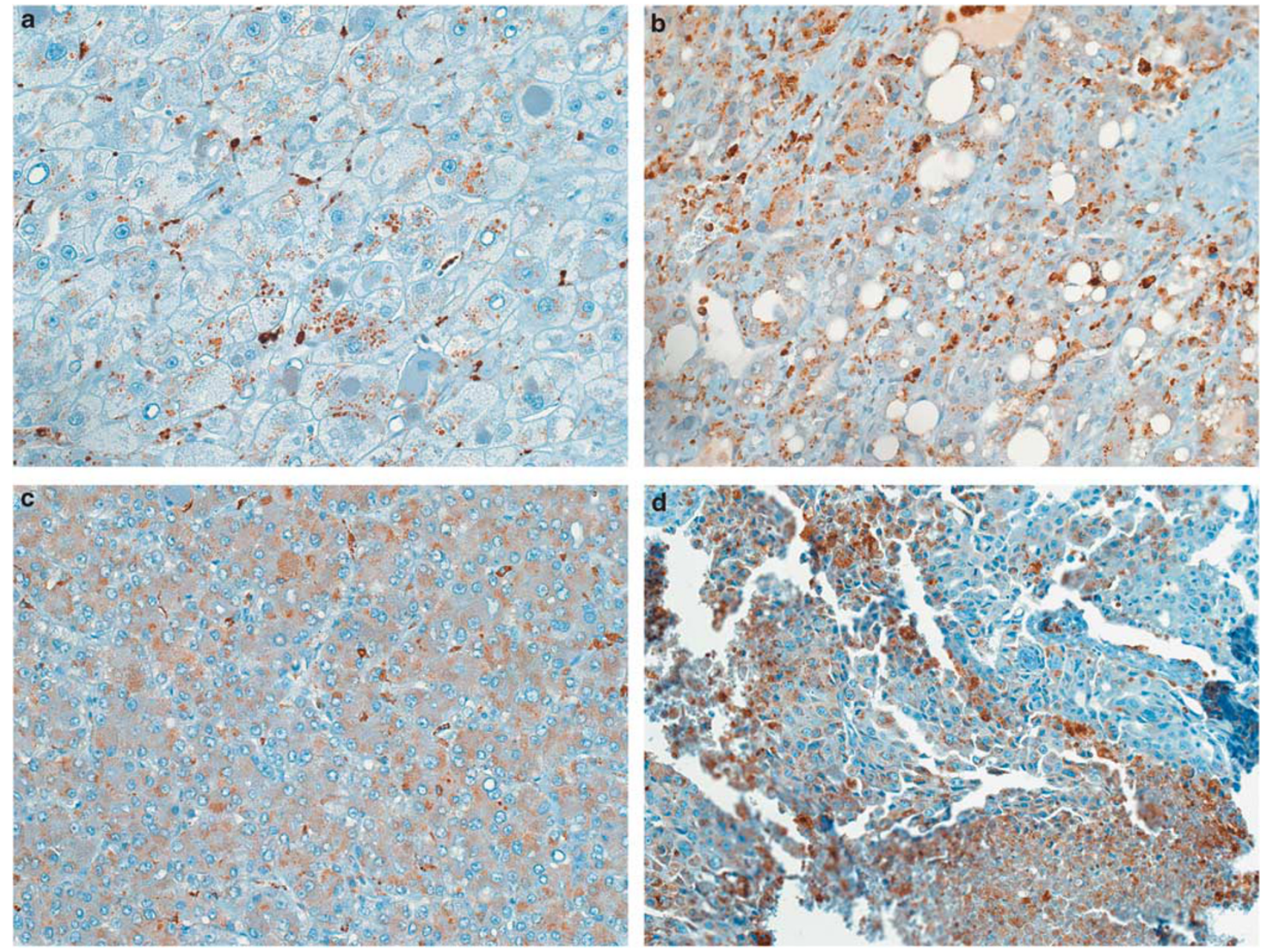

Figure 2 Typical hepatocellular carcinomas were positive for CD68 staining in some cases. (a) CD68, original magnification, $\times 100$ : a typical hepatocellular carcinoma shows a stippled or granular staining pattern that closely resembles fibrolamellar carcinoma. (b) CD68, original magnification, $\times 100$ : typical hepatocellular carcinomas with fatty change were most likely to show a CD68 staining pattern similar to fibrolamellar carcinoma. (c) CD68, original magnification, $\times 100$ : In rare cases, there was a more diffuse CD68 staining pattern. (d) CD68, original magnification, $\times 100$ : CD68 positivity could also be seen focally near areas of tumor necrosis.

Table 2 Accuracy indices of CD68 staining as a marker for the diagnosis of fibrolamellar carcinoma in a primary liver carcinoma ${ }^{\mathrm{a}}$

\section{Performance characteristics}

Value (\%)

Sensitivity

Specificity

Positive predictive value

Negative predictive value

95.6

80.3

62.9

98.1

${ }^{\mathrm{a} C a l c u l a t e d}$ using 23 cases with primary fibrolamellar carcinoma and 66 cases with typical hepatocellular carcinoma.

showed CD68 staining. Whether this represents dysregulation of similar biological pathways is unclear. Also of note, CD68 positivity was seen adjacent to necrosis and CD68 staining in this context should be interpreted cautiously. Another point that bears emphasis is that none of the cases in the control groups that were CD68 positive had the typical histological features of fibrolamellar

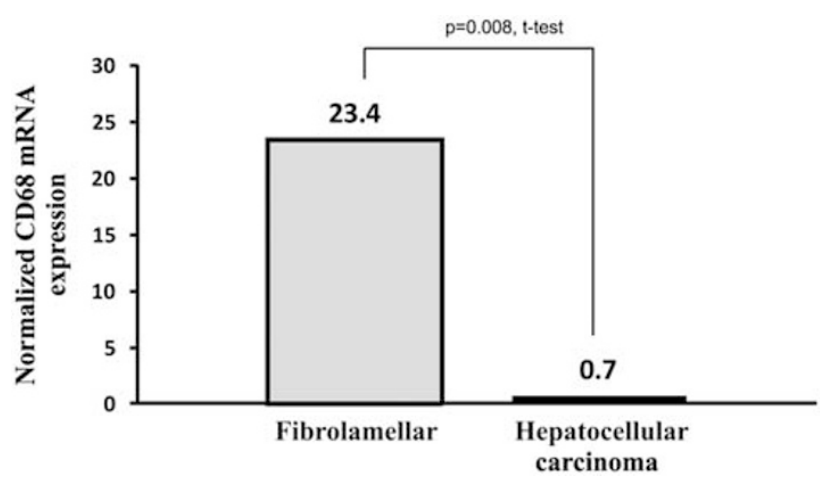

Figure 3 Fibrolamellar carcinomas $(N=7)$ showed significantly more expression of CD68 than typical hepatocellular carcinomas $(N=6)$. The typical hepatocellular carcinomas arose in livers with cirrhosis and chronic hepatitis C.

carcinoma. Thus, combining the histological findings with CD68 would likely increase the specificity of CD68 staining for fibrolamellar carcinoma. 
We propose that the reproducibility of the histological diagnosis of fibrolamellar carcinoma can be substantially improved by careful attention to the full pattern of histological features (large polygonal tumor cells with abundant eosinophilic cytoplasm, large vesiculated nuclei, large nucleoli, and lamellar fibrosis) $^{1}$ and that most cases should be CK7 positive $^{5,8,9}$ as well as CD68 positive. In the absence of CK7 and/or CD68 positivity, a diagnosis of fibrolamellar carcinoma should be carefully re-considered.

These findings also extend our understanding of the biology of fibrolamellar carcinomas. Their oncocytic appearance has typically been attributed to cytoplasmic swelling by numerous packed mitochondria, as seen in electron microscopy studies. The current study indicates that fibrolamellar carcinomas also have increased lysosomal or endosomal accumulations in their cytoplasm, which may suggest that abnormalities in endosomal/lysosomal trafficking are characteristic of fibrolamellar carcinomas. Overexpression occurs at the level of both mRNA and protein.

Of note, one previous study examined a case of fibrolamellar carcinoma and found it to be CD68 positive when using clone KP1 but not clone PG-M1. ${ }^{10}$ They also reported the fibrolamellar carcinoma was weakly positive for Ki-M1P and $3 \mathrm{~A} 5$, and negative for and anti-lysozyme. ${ }^{10}$ These authors also reported that the non-neoplastic liver in a case of biliary atresia was CD68 positive (using clone KP1) and that, based on electron microscopy, immunoreactivity correlated with lysosome-like granules as well as electron dense structures that were probably bile. Thus, some caution is likely warranted when staining a tumor rich in cytoplasmic bile for CD68, as the bile may be stored in lysosomes within tumor cells.

In sum, CD68 immunostaining is a sensitive marker for fibrolamellar carcinoma that may be of use in routine diagnostic surgical pathology. In addition, it may be of use in research studies by helping properly classify cases of fibrolamellar carcinoma. This is of importance because it can help ensure a uniform biological entity is being investigated by different groups, as the incorporation of non-fibrolamellar carcinoma cases in such studies is unlikely to improve our understanding of the biology of this disease.

\section{Acknowledgements}

We gratefully acknowledge the support of the National Institutes of Health, R01DK078686 (MT), the Fibrolamellar Cancer Foundation, and the International Cancer Alliance (ICARE).

\section{Disclosure/conflict of interest}

The authors declare no conflict of interest.

\section{References}

1 Torbenson M. Review of the clinicopathologic features of fibrolamellar carcinoma. Adv Anat Pathol 2007;14: 217-223.

2 Malouf G, Falissard B, Azoulay D, et al. Is histological diagnosis of primary liver carcinomas with fibrous stroma reproducible among experts? J Clin Pathol 2009;62:519-524.

3 El-Serag HB, Davila JA. Is fibrolamellar carcinoma different from hepatocellular carcinoma? A US population-based study. Hepatology 2004;39:798-803.

4 Vivekanandan P, Micchelli ST, Torbenson M. Anterior gradient-2 is overexpressed by fibrolamellar carcinomas. Hum Pathol 2009;40:293-299.

5 Klein WM, Molmenti EP, Colombani PM, et al. Primary liver carcinoma arising in people younger than 30 years. Am J Clin Pathol 2005;124:512-518.

6 Kannangai R, Vivekanandan P, Martinez-Murillo F, et al. Fibrolamellar carcinomas show overexpression of genes in the RAS, MAPK, PIK3, and xenobiotic degradation pathways. Hum Pathol 2007;38:639-644.

7 Biosystems A. Guide to performing relative quantitation of gene expression using real-time quantitative PCR. http://www3.appliedbiosystems.com/cms/ groups/mcb_support/documents/generaldocuments/ cms_042380.pdf, 2010.

8 Abdül-Al HM, Wang G, Makhlouf HR, et al. Fibrolamellar hepatocellular carcinoma: an immunohistochemical comparison with conventional hepatocellular carcinoma. Int J Surg Pathol 2010;18:313-318.

9 Ward SC, Huang J, Tickoo SK, et al. Fibrolamellar carcinoma of the liver exhibits immunohistochemical evidence of both hepatocyte and bile duct differentiation. Mod Pathol 2010;23:1180-1190.

10 Kaiserling E, Ruck P, Xiao JC. Immunoreactivity of neoplastic and non-neoplastic hepatocytes for CD68 and with 3A5, Ki-M1P, and MAC387 light and electron microscopic findings. Int Hepatol Commun 1995;3: 322-329. 